\title{
Malignant ascites - current treatment and novel therapeutic options
}

\author{
A. Stange \\ National Center for Tumor Diseases Heidelberg, Heidelberg, Germany
}

Received 1 November 2011; accepted 23 February 2012

\begin{abstract}
Malignant ascites is a common problem in patients with advanced malignancies and peritoneal spread of tumour. Treatment strategies include paracentesis, diuretics and peritoneovenous shunts; however, there are no established evidence-based guidelines for optimal therapy. This review is intended to add clarity to the current procedures for the management of malignant ascites, and furthermore discusses new promising approaches.
\end{abstract}

Keywords: Ascites, peritoneal carcinomatosis, paracentesis, diuretics, peritonevenous shunt.

\section{Introduction}

Malignant ascites is a common problem in advanced neoplasms and occurs especially in association with breast, bronchus, ovary, stomach, pancreas and colorectal cancer [1]. Up to $20 \%$ of all patients with malignant ascites have tumours of unknown primary origin $[1,2]$. The onset and progression of malignant ascites is associated with a rapid deterioration in quality of life and a poor prognosis. Overall survival is mainly determined by the origin of the primary cancer. Patients with ovarian cancer have a better prognosis while patients with malignant ascites of gastrointestinal origin or unknown origin have the worst outcome [3]. Large amounts of ascites can induce increased abdominal pressure and thereby cause discomfort and distress with symptoms such as abdominal pain (53\%), nausea (37\%), anorexia (36\%), vomiting (25\%), fatigue (17\%), dyspnoea (11\%) and early fullness (6\%) [3]. Especially in end stage disease treatment is aimed at improving quality of life by achieving symptom relief with minimal invasive techniques that at the same time have the lowest risk for complications.

\section{Pathophysiology and diagnosis}

Pathophysiology of malignant ascites is multifactorial and yet incompletely understood. In addition to decreased

Correspondence: Annika Stange, MD, National Center for Tumor Diseases Heidelberg, Im Neuenheimer Feld 460, 69120 Heidelberg, Germany.

E-mail: annika.stange@med.uni-heidelberg.de lymphatic drainage and hormonal mechanisms cytokinemediated increased capillary permeability is discussed to play a role, since malignant ascites is usually protein-rich [4]. Mediators such as vascular endothelial growth factor (VEGF), interleukin-6 and tumour necrosis factor may play a role as well [5]. In individual cases hypoalbuminemia due to impaired liver function second to liver metastases or portal hypertension caused by large liver tumours occluding portal or hepatic veins may contribute to ascites as well. After diagnosis by physical examination and imaging malignant ascites is usually confirmed by diagnostic paracentesis. Cytological analysis is the most specific test to demonstrate malignant ascites. It is about $97 \%$ sensitive with peritoneal carcinomatosis [6], but is poor in detecting other types of malignant ascites. Cell counts with a differential are useful in the presumptive diagnosis of bacterial peritonitis, particularly if the neutrophil count is greater than 250 cells per ml. Nevertheless peritoneal carcinomatosis can mimic spontaneous bacterial peritonitis. If infection is suspected, a Gram stain and culture should be performed. The serum-ascites albumin gradient (SAAG) is recommended for the differential diagnosis and management of ascites. SAAG is calculated by subtracting the ascitic fluid albumin level from the serum level obtained on the same day. A gradient of more than $1.1 \mathrm{~g} /$ $\mathrm{dL}$ indicates presence of portal hypertension, a decreased gradient $(<1.1 \mathrm{~g} / \mathrm{dL})$ is found in peritoneal carcinomatosis [7]. Additional imaging (e.g. Doppler ultrasound of the portal vein) may help to specify the cause of ascites in individual cases.

\section{Therapeutic options}

Surveys of practices in management of malignant ascites from England [3] and Canada [8] show that paracentesis, diuretics and systemic chemotherapy against the underlying malignancy are commonly used procedures. Peritoneovenous shunts, cytoreductive surgery and (hyperthermic) intraperitoneal (i.p.) chemotherapy are used as well. However, in contrast to the well-established guidelines for treatment of the origin of the primary cancer, there are no evidence-based guidelines for optimal therapy of malignant ascites. Current approaches are mainly based on personal experience and adapted from the treatment of cirrhosis-associated ascites. 


\section{Therapeutic paracentesis and permanent drains}

In about $90 \%$ of patients therapeutic paracentesis yields good, although temporary relief of symptoms. A review of studies showed no consensus on the rate or maximum volume of fluid withdrawal. Reported complications are infrequent and include hypotension, pulmonary embolism, secondary peritonitis and perforation. Severe hypotension and renal impairment might be reduced by concurrent volume expansion. Studies in patients with cirrhosis-associated ascites showed that in paracentesis of large volume albumin is superior to other plasma expanders in preventing circulatory dysfunction. Therefore, infusion of albumin (e.g., 6-8 g per liter of ascites removed) has been used concurrent to paracentesis of malignant ascites. However, the need for colloid replacement remains controversial since in the context of malignant ascites no trials have been performed. Clinical experience suggests that intravenous albumin infusion is not generally necessary. In a retrospective analyses of 30 paracenteses in 12 patients with malignant ascites up to $5 \mathrm{~L}$ fluid could be withdrawn and intravenous fluids were only given when specificallyindicated. There was no case of symptomatic hypotension and blood products or intravenous fluids were given in only 6 cases [9]. According to a prospective study observing 48 paracenteses in 44 patients, a mean withdrawal of $5.3 \mathrm{~L}$ (range 0.8-15 L, median $4.9 \mathrm{~L}$ ) is needed in order to achieve a significant symptom relief [10]. No severe side effects were reported and patients did not require volume expansion.

If serial paracentesis does not yield fluid control permanent drains can be considered. For non-tunnelled catheters (e.g. pigtail catheter) a complication rate of up to $30 \%$ has been reported, including infection, sepsis and occlusion. In contrast, a retrospective series of 40 tunnelled catheters reported low complication rates which were comparable to repeated large volume paracentesis in 67 patients [11].

\section{Diuretic treatment}

Diuretics are often used in the management of malignant ascites [7], despite their use being highly controversial. There are no randomised controlled trials on efficacy or effectiveness.

Becker and colleagues evaluated 5 studies including 113 patients with different tumours and found diuretics to be successful in approximately $43 \%$ [12]. However, phase II data [13] suggest that response to diuretics is restricted to patients with a SAAG $>1.1 \mathrm{~g} / \mathrm{dL}$ (congruent to benign ascites due to liver cirrhoses), whereas malignant ascites with a SAAG $<1.1 \mathrm{~g} / \mathrm{dL}$ is highly resistant to diuretic use. Some authors even state that medical therapies, such as diuretics as well as sodium and fluid restriction, are not effective in most oncological patients independent from SAAG [14]. It has to be emphasized that patients and doctors should be aware of possible side effects such as hypovolemia and renal failure when using diuretics.

\section{Octreotide}

Somatostatin and octreotide are often used successfully to treat diarrhoea and lymphatic leakage due to abdominal and thoracic surgery. Case reports suggest that subcutaneous octreotide is also effective in the management of chylous ascites in malignant disease [15]. Data from a Phase III, randomised, double-blind, placebo-controlled, multicentre study evaluating the efficacy in a broad range of tumours which started in 2005 are pending.

\section{Peritoneovenous shunts}

Initially developed for use in patients with refractory ascites due to liver cirrhoses peritoneovenous shunts subsequently became popular in the management of malignant ascites in Anglo-American countries. Relevant contraindications are loculated ascites, portal hypertension, coagulation disorders, and advanced cardiac or renal impairment. Furthermore due to higher risk of shunt occlusion haemorrhagic ascites and fluid protein content $>4.5 \mathrm{~g} / \mathrm{L}$ are considered as contraindications to shunt placement. Patients with ovarian and breast cancers who undergo peritoneovenous shunting have the best response rates $(>50 \%)$ compared to gastrointestinal cancers (10-15\%) [4]. Reported side effects include pulmonary oedema or embolism, subclinical as well as clinically relevant disseminated intravascular coagulation, and infection. These complications have to be expected in about $6 \%$ of patients [12]. Even though systemic dissemination of malignant cells is theoretically obligatory, postmortem analysis proved this concern to be clinically insignificant [4]. However, shunting is not an established procedure in managing malignant ascites in Europe, possibly due to balancing benefit and potential risks differentially.

\section{Transjugular intrahepatic portosystemic shunt}

For patients with cirrhosis and refractory ascites who have relatively good hepatic and renal function, transjugular intrahepatic portosystemic shunt (TIPS) is considered the treatment of choice. In two cases of malignant portal and hepatic vein occlusion, TIPS improved ascites and quality of life [16]. Thus in selected cancer patients with metastatic disease to the liver or locally advanced cancer, e.g. biliary cancer, TIPS can be considered. As with any palliative management option, the decision to pursue invasive procedures is dependent on the patient's goals in the context of the disease.

\section{Intraperitoneal chemotherapy and cytoreductive surgery}

The intent of i.p. therapy in malignant ascites is usually palliative. I.p. chemotherapy as well as hyperthermic i.p. chemotherapy (HIPEC), which is proven to have enhanced cytotoxicity, have been investigated in small series and must be considered experimental [17].

The objective of cytoreductive surgery in combination with HIPEC is to remove all macroscopic tumour after abdominal exploration leaving only microscopic residual disease for improved tumour tissue penetration with HIPEC [18]. This multimodal approach has been shown to improve survival in appropriately selected patients and is mainly applied to patients with metastastic appendiceal or colorectal cancer limited to the peritoneum $[18,19]$.

\section{Intraperitoneal monoclonal antibodies}

Catumaxomab is a trifunctional bispecific antibody that binds to tumour cells expressing human epithelial cell adhesion 
Malignant ascites

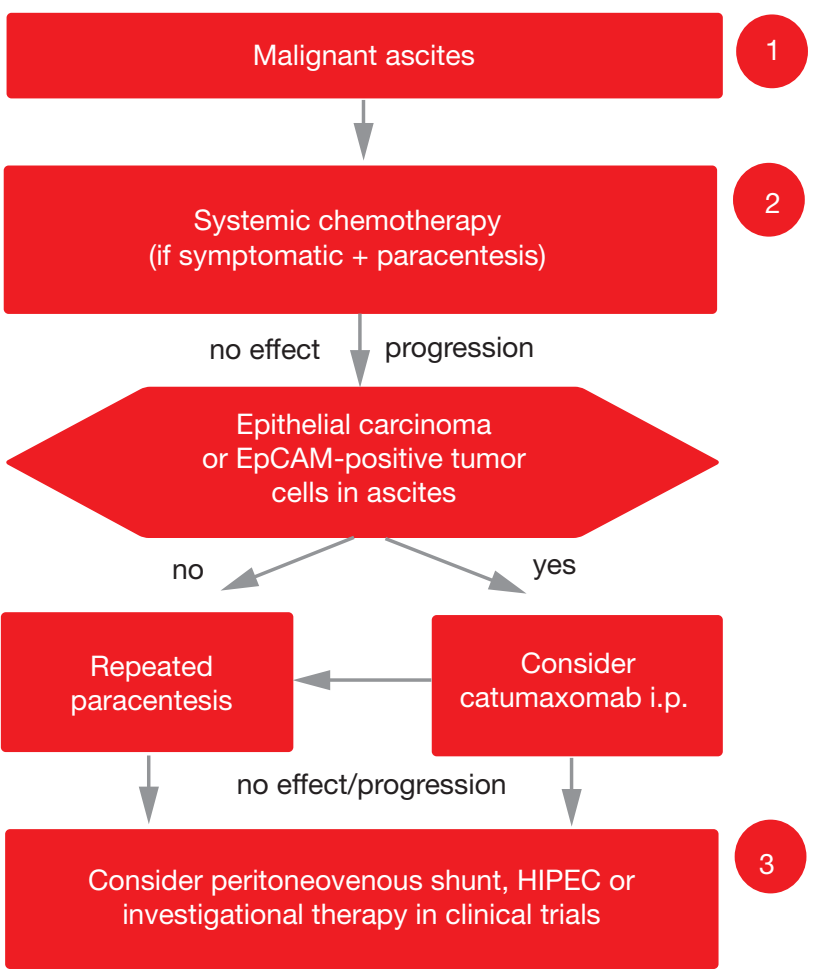

Fig. 1: Algorithm for diagnosis and treatment of malignant ascites. 1: Diagnostic includes ultrasound, cytology, SAAG, 2: Consider diuretics, especially if SAAG $>1.1 \mathrm{~g} / \mathrm{dL}$, 3: HIPEC must be considered experimental and can only be applied at specialized centres

molecule (EpCAM), known to be expressed in gastric, hepatobiliary, colonic, and other epithelial carcinomas [17]) and redirects CD3+ T lymphocytes and Fc $\gamma$-receptor-positive accessory tumour cells such as macrophages, dendritic cells and natural killer cells to malignant cells [21]. Based on preclinical data simultaneous activation of $\mathrm{T}$ cells and accessory immune cells induces a variety of immunological events that ultimately lead to tumour cell elimination by different killing mechanisms such as antibody-dependent cellular cytotoxicity (ADCC), phagocytosis and perforine-dependent lysis [22, 23]. In an international Phase II/III study 258 cancer patients with recurrent symptomatic malignant ascites resistant to conventional chemotherapy were randomised to paracentesis plus catumaxomab administered as an i.p. infusion on days $0,3,7$ and 10 or paracentesis alone. Independent of the type of tumour the puncture free survival was significantly longer in the catumaxomab group (median 46 days) than the control group (median 11 days) (hazard ratio $=0.254$ : $p<0.0001$ ) [24]. Related to the immunological mode of action the most commonly reported adverse events are cytokine release-related symptoms (pyrexia, nausea and vomiting), which are generally mild to moderate and manageable by standard symptomatic treatment [24]. Catumaxomab has been approved in the European Union since April 2009 for the i.p. treatment of malignant ascites in patients with EpCAM-positive carcinomas where standard therapy of the underlying malignancy is not available or no longer feasible.

Since VEGF is thought to promote ascites by increasing vascular permeability, preclinical data and reported small series from patients treated off label i.p. with the anti-VEGF antibody bevacizumab support the hypothesis that targeting VEGF may have the potential to prevent local fluid accumulation [25]. Therefore, the i.p. application of bevacizumab is currently being investigated in a randomised Phase II trial.

\section{Other investigational therapies}

A recently published Phase II study evaluated the treatment with intravenous aflibercept in 16 patients with advanced chemo-resistant ovarian cancer and symptomatic malignant ascites. The fusion protein, designed to bind to VEGF-A, VEGF-B, and placental growth factor (PIGF) significantly reduced the interval between repeat paracenteses. The safety profile was consistent with that reported for anti-VEGF agents [26].

Up to now further novel approaches have only been studied only in small series. Improvement in ascites is reported in response to cytokine therapy with i.p. $\alpha$ or $\beta$ interferon, tumour necrosis factor $\alpha$ or infectious agents such as intracavitary Corynebacterium parvum. Also i.p. gold isotope (198Au), chromic phosphate colloid (32ChrP) and matrix metalloproteinase inhibitors were investigated in Phase I/II trials [11]. Taken together, these treatments must be considered as highly experimental and should not be applied outside clinical trials.

\section{Conclusion}

The management of malignant ascites is a significant challenge in medical oncology. Although diuretics, paracentesis, peritoneal drains and venous shunts are widely used procedures, evidence is weak and randomised controlled trials identifying optimal therapy are lacking. Newer therapies are emerging and await further study. The i.p. application of the approved trifunctional antibody catumaxomab seems to be a promising approach, its successful implementation in daily clinical practice has nevertheless to be proven in the future. Since no evidence-based guidelines exist, the different treatment options should be applied with the goal of palliation of symptoms that is best suited for the individual patient.

\section{Take home message}

Repeated therapeutic paracenteses yield good, although temporary relief of symptoms. Available data about diuretics are conflicting. Intraperitoneal monoclonal antibodies are a new promising approach.

\section{Conflict of interest}

The author declares that there is no conflict of interest.

\section{References}

[1] Runyon BA. Care of patients with ascites. N Engl J Med, 330: 337-42, 1994.

[2] Ringenberg QS, Doll DC, Loy TS, et al. Malignant ascites of unknown origin. Cancer, 64: 753-5, 1989.

[3] Ayantunde AA, Parsons L. Pattern and prognostic factors in patients with malignant ascites: a retrospective study. Ann Oncol, 18: 945-9, 2007.

[4] Adam RA, Adam YG. Malignant ascites: past, present, and future. J Am Coll Surg, 198: 999-1011, 2004.

[5] Smith EM, Jayson GC. The current and future management of malignant ascites. Clin Oncol, 15: 59-72, 2003. 
[6] Runyon BA, Hoefs JC, Morgan TR. Ascitic fluid analysis in malignancy-related ascites. Hepatology, 8: 1104-9, 1988.

[7] Runyon BA, Montano AA, Akriviadis EA, et al. The serum-ascites albumin gradient is superior to the exudate-transudate concept in the differential diagnosis of ascites. Ann Intern Med, 117: 215-20, 1992.

[8] Lee CW, Bociek G, Faught W. A survey of practices in management of malignant ascites. J Pain Symptom Manage, 16: 96-101, 1998.

[9] Stephenson J, Gilbert J. The development of clinical guidelines on paracentesis for ascites related to maglinancy. Palliat Med, 16: 213-8, 2002.

[10] McNamara P. Paracentesis - an effective method of symptom control in the palliave setting? Palliat Med, 14: 62-4, 2000.

[11] Rosenberg S, Courtney A, Nemcek AA, et al. Comparison of percutaneus management techniques for recurrent maglinant ascites. J Vasc Interv Radiol, 15: 1129-31, 2004.

[12] Becker G, Galandi D, Blum HE. Malignant ascites. Systemic review and guideline for treatment. Eur J Cancer, 42: 589-97, 2006.

[13] Pockros PJ, Esrason KT, Nguyen C, et al. Mobilization of malignant ascites with diuretics is dependent on ascitic fluid characteristics. Gastroenterology 103: 1302-6, 1992.

[14] Rosenberg SM. Palliation of malignant ascites. Gastroenterol Clin North Am, 35(1): 189-99, xi, 2006.

[15] Mincher L, Evans J, Jenner MW, et al. The successful treatment of chylous effusions in malignant disease with octreotide. Clin Oncol (R Coll Radiol), 17(2): 118, 2005.

[16] Burger JA, Ochs A, Wirth K, et al. The transjugular stent implantation for the treatment of malignant portal and hepatic vein obstruction in cancer patients. Ann Oncol, 8: 200-2, 1997.

[17] Markman M. Intraperitoneal antineoplastic drug delivery: rationale and results. Lancet Oncol, 4(5): 277-83, 2003.

[18] Verwaal VJ, Bruin S, Boot H, et al. 8-year follow-up of randomized trial: cytoreduction and hyperthermic intraperitoneal chemothera- py versus systemic chemotherapy in patients with peritoneal carcinomatosis of colorectal cancer. Ann Surg Oncol, 15: 2426-32, 2008.

[19] Elias D, Lefevre JH, Chevalier J, et al. Complete cytoreductive surgery plus intraperitoneal chemohyperthermia with oxaliplatin for peritoneal carcinomatosis of colorectal origin. J Clin Oncol, 27: 681-5, 2009.

[20] Went PT, Lugli A, Meier S, et al. Frequent EpCam protein expression in human carcinomas. Hum Pathol, 35: 122-8, 2004

[21] Bokemeyer C. Catumaxomab-trifunctional anti-EpCAM antibody used to treat malignant ascites. Expert Opin Biol Ther, 10(8): 125969, 2010.

[22] Zeidler R, Reisbach G, Wollenberg B, et al. Simultaneous activation of $T$ cells and accessory cells by a new class of intact bispecific antibody results in efficient tumor cell killing. J Immunol, 163: 1246-52, 1999.

[23] Zeidler R, Mysliwietz J, Csánady M. The Fc-region of a new class of intact bispecific antibody mediates activation of accessory cells and NK cells and induces direct phagocytosis of tumour cells. Br J Cancer, 83: 261-6, 2000

[24] Heiss MM, Murawa P, Koralewski P, et al. The trifunctional antibody catumaxomab for the treatment of malignant ascites due to epithelial cancer: results of a prospective randomized phase II/III trial. Int J Cancer, 127(9): 2209-21, 2010.

[25] Kobold S, Hegewisch-Becker S, Oechsle Ket al. Intraperitoneal VEGF inhibition using bevacizumab: a potential approach for the symptomatic treatment of malignant ascites? Oncologist, 14(12): 1242-51, 2009.

[26] Colombo N, Mangili G, Mammoliti S, et al. A phase II study of aflibercept in patients with advanced epithelial ovarian cancer and symptomatic malignant ascites. Gynecol Oncol, 125(1): 42-7, 2012. 